\title{
Current Challenges and Future Opportunities for Child and Adolescent Psychiatry in Japan
}

\author{
Masaru Tateno ${ }^{1,2} \otimes$, Takahiko Inagaki $^{3,4}$, Takuya Saito ${ }^{5}$, Anthony P. S. Guerrero ${ }^{6}$, and Norbert Skokauskas ${ }^{7}$ \\ ${ }^{1}$ Tokiwa Child Development Center, Tokiwa Hospital, Sapporo, Japan \\ ${ }^{2}$ Department of Neuropsychiatry, Sapporo Medical University, School of Medicine, Sapporo, Japan \\ ${ }^{3}$ Department of Psychiatry, Shiga Prefectural Mental Medical Center, Shiga, Japan \\ ${ }^{4}$ Department of Psychiatry, Shiga University of Medical Science, Shiga, Japan \\ ${ }^{5}$ Department of Child and Adolescent Psychiatry, Graduate School of Medicine, Hokkaido University, Sapporo, Japan \\ ${ }^{6}$ Department of Psychiatry, Child and Adolescent Psychiatry Division, University of Hawai'i John A. Burns School of Medicine, Honolulu, HI, USA \\ ${ }^{7}$ Centre for Child and Adolescent Mental Health and Child Protection Faculty of Medicine, Trondheim, Norway
}

\begin{abstract}
Japan has been facing a serious shortfall of child and adolescent psychiatric workforce relative to increasing service needs. Likely because of a combination of limited workforce supply and limited trust or perception of effectiveness, mental health services are under-utilized by the educational and child welfare systems. Child and adolescent psychiatry (CAP) has not been a formally established specialty in Japan. The lack of basic structure in the specialty most likely contributes to a lack of training facilities, limited exposure to and interest in the specialty, and hence an inadequate workforce. To date, there exists no standardized training program for CAP in Japan and each training hospital determines its own teaching curriculum and training content. Clinical experience in CAP varies greatly among hospitals. To solve current problems in child and adolescent psychiatry in Japan, we advocate for the development and establishment of a more standardized child and adolescent psychiatry training system that is akin to what exists in the US and that teaches and evaluates according to specific competencies. Through standardizing care and education and ultimately improving workforce, the quality of mental health services can be raised. The tragic and costly consequences of unidentified and untreated mental illness in youth can be avoided by taking timely evidence based actions in partnership with others.

Psychiatry Investig 2017;14(5):525-531
\end{abstract}

Key Words Child and adolescent psychiatry, Education, Mental health service, Post-graduate training.

\section{INTRODUCTION}

Japan has been facing a serious shortfall of child and adolescent psychiatric workforce relative to service needs. ${ }^{1,2} \mathrm{Re}-$ cent large scale epidemiological studies have demonstrated that the prevalence rate of neurodevelopmental disorders, such as Autism Spectrum Disorder (ASD) and Attention Deficit Hyperactivity Disorder (ADHD), continues to increase. ${ }^{3-6}$ This trend is similarly observed in Japan. ${ }^{7-9}$ According to the World Health Organization, approximately $10-20 \%$ of youth experience mental health disorders. ${ }^{10}$ Furthermore, $70 \%$ of mental

Received: August 3, 2016 Revised: November 8, 2016

Accepted: November 30, 2016 Available online: July 26, 2017

$\square$ Correspondence: Masaru Tateno, MD, PhD

Tokiwa Child Development Center, Tokiwa Hospital, Tokiwa 3-1-6-1, Minamiku, Sapporo 005-0853, Japan

Tel: +81-11-591-4711, Fax: +81-11-591-0922, E-mail: tatema@sapmed.ac.jp

(c) This is an Open Access article distributed under the terms of the Creative Commons Attribution Non-Commercial License (http://creativecommons.org/licenses/bync/4.0) which permits unrestricted non-commercial use, distribution, and reproduction in any medium, provided the original work is properly cited. health disorders have their onset prior to the age of 25 years. ${ }^{11}$ The Sequenced Treatment Alternatives to Relieve Depression $\left(\mathrm{STAR}^{*} \mathrm{D}\right)$ study ${ }^{12,13}$ revealed that the onset of Major Depression was earlier than previously presumed, with the first episode most frequently occurring in adolescence. ${ }^{14}$ Untreated mental health problems in childhood can be transmuted into various psychiatric diseases. ${ }^{15-20}$ Moreover, there are several psychiatric disorders (i.e., early onset neurodevelopmental disorders) that are most often diagnosed in childhood and that require early interventions. ${ }^{21,22}$ There is growing awareness of the importance of early intervention in psychosis, especially in adolescents. ${ }^{23,24}$ Recently, internet overuse has become a serious problem among adolescents in Asian countries. ${ }^{25-29}$ While Japan succeeded in reducing the total number of completed suicides to less than 300,000 per year, ${ }^{30}$ the rate of suicide in teenagers was unchanged. ${ }^{31-33}$ One of the reasons for the continued high adolescent suicide rate might be the widespread and growing use of smartphones that could serve as a vehicle for on-line or cyber-bullying. ${ }^{34,35}$ School refusal (futoukou in 
Japanese) is a problem that is relatively unique in Japan and that often requires medical intervention. ${ }^{36-40}$ A nationwide survey conducted by the Ministry of Education, Culture, Sports, Science and Technology (MECSST) in 2015 reported that the rate of school refusal was $2.76 \%$ among junior high school students in Japan. ${ }^{41}$ In this context, MECSST defined school refusal as a protracted school absence (without documented need to receive medical care), typically more than 30 days per year, caused by psychological factors such as fear, anxiety, anger, and sense of refusal. Continuous avoidance of social situations in school may result in severe social withdrawal, named hikikomori, in adulthood. ${ }^{42-49}$ All of these mental health problems typically occur in childhood or adolescence and increase societal demands for child and adolescent psychiatry services in Japan.

\section{AIM AND METHODS OF THIS REVIEW}

The authors of this review paper are primary and supporting members of the Consortium on Academic Child and Adolescent Psychiatry in the Far East (CACAP FE). ${ }^{50}$ The multinational survey conducted by CACAP FE revealed that, although Japan has advanced medical technology and an overall high quality of care in medicine, CAP remained unrecognized as an established subspecialty, and there were no national guidelines for CAP training. To address the significant shortages of youth mental health services in Japan, the authors chose to further describe, via this paper, the underlying challenges and potential solutions.

Because CAP appears to have had the longest history of establishment as a specialty in the United States and Europe, and also because Japan has been significantly influenced by training and service models from these places, we chose to also include, in this review, and update on the current status of CAP training and services in the United States and Europe.

\section{CURRENT GAPS IN CHILD AND ADOLESCENT MENTAL HEALTH SERVICES IN JAPAN}

The $2.76 \%$ among junior high school students in Japan translates into 95,000 youth, excluding those identified with an illness. Furthermore, $68.4 \%$ of these youth stay out of for over one year. ${ }^{41}$ The students included in these high numbers are not receiving treatment-notwithstanding the fact that they had reasons to withdraw from school. It is clear that better prevention programs are needed to address the issue.

Furthermore, in Japan, the "school counselor" is deployed in over $75 \%$ of all junior high schools. ${ }^{51}$ Their defined role is to optimize child welfare by establishing a connection between schools and mental health services. In view of this fact, it appears that the rate of withdrawn students who do not receive mental health services-notwithstanding the existence of a theoretical mechanism to facilitate referrals-is unacceptably high.

Bullying is a serious concern identified in Japanese schools. ${ }^{49,52-54}$ The Japanese government reported that the number of bullying incidents was about $188,000 .{ }^{55}$ Although the victims are provided with support, no interventions for the perpetrator exist. There likely are insufficient child and adolescent mental health service staff. Public education is not enough to counteract this serious and growing mental health problem.

In Shiga Prefecture, which is fairly representative of other prefectures, welfare agencies were consulted for over 5,000 children and adolescents in 2014. Among these youth, only $7 \%$ had consulted with mental health services, and only an additional $2 \%$ had been encouraged to consult with mental health services but had not yet done so. ${ }^{56}$ It appears that the system of cooperation between welfare and mental health services is not effective, as illustrated in Figure 1.

In addition to stigma in child and adolescent mental health services, ${ }^{57,58}$ child welfare agencies seem not to trust them, and likewise, there are not enough mental health providers to build and solidify trust through their services. In child welfare agencies' impression, following referrals to mental health services, the rate of "improved and the problem was solved" as an out-

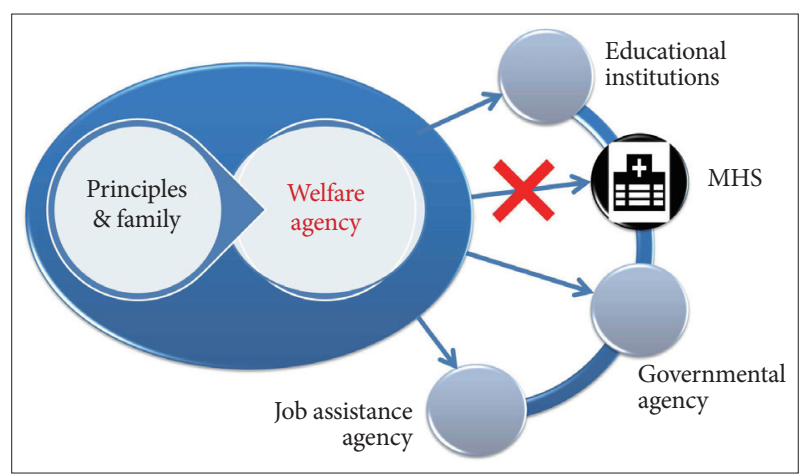

Figure 1. Failure of cooperation between welfare and mental health service (MHS).

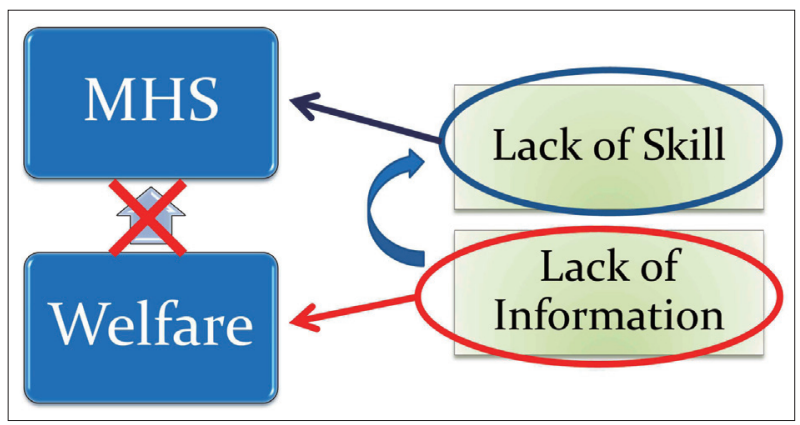

Figure 2. Lack of information diminishes cooperation between welfare and MHS. MHS: mental health service. 
come is only $18 \%$, and the cumulative rate of "no change" and "worsened" as outcomes is $38 \%{ }^{56}$

It appears that, currently in Japan, the quality and accessibility of mental health services are not sufficient, and due to a lack of information, there is a fundamental misunderstanding of mental health services' role and a lack of skill development in this area (Figure 2).

\section{CHILD AND ADOLESCENT PSYCHIATRIC TRAINING AND WORKFORCE CHALLENGES IN JAPAN}

Unfortunately, child and adolescent psychiatry (CAP) has not been a formally established specialty in Japan. CAP is considered a subspecialty of Psychiatry and Pediatrics. ${ }^{1,59}$

To date, there exists no standardized training program of CAP in Japan. Because each training hospital determines its own teaching curriculum, training content and clinical experience in CAP varies greatly among hospitals. However, there are several academic societies related to CAP that have respective certification systems for specialist in CAP: The Japanese Society for Child and Adolescent Psychiatry (JSCAP), Japanese Society for Adolescent Psychiatry (JSAP), Japanese Society of Pediatric Psychiatry and Neurology (JSPPN), and Japanese Society of Psychosomatic Pediatrics (JSPP).

JSCAP certification requirements include: more than 5 years of clinical experience in medicine, including over two years in general psychiatry and over three years in CAP; submission of a list of 30 CAP cases seen in the preceding three years; and three case reports. The clinician certified by the JSCAP is regarded as a specialist in CAP in Japan when statistical data on the CAP workforce are presented.

According to data from the Japanese Ministry of Health, Labor and Welfare, about $5 \%$ of all medical doctors (about 300,000 in total) are psychiatrists. Among them only 289 are JSCAP-certified CAP specialists (JSCAP website; as of May 10, 2016). Japan, with a total population exceeding 120 million people, is facing a serious shortfall of child psychiatrists, despite increasing social demands and a need to increase the number of clinicians who deal with children's mental health problems. Clearly, there is a need to produce and retain a child and adolescent psychiatric workforce that can fully respond to current mental health needs.

As summarized by Hirota and colleagues, ${ }^{50}$ recognition of CAP as an official subspecialty and existence of systematized training did not necessarily depend on income levels among countries in Far East Asia. For example, at the time of writing, there were no national guidelines for CAP training in such high income countries as Japan and Singapore. ${ }^{1,60}$

Unfortunately, in Japan, the lack of basic structure in the specialty most likely contributes to lack of training facilities, lack of an adequate workforce, off-label use of medications, and ultimately lack of access to child and adolescent mental health services. Clearly, the training and certification system does not reflect the needs of the public.

The Japanese Society of Psychiatry and Neurology (JSPN) started a specialist accreditation system in 2006. ${ }^{61-63}$ The requirements for application are as follows: 1) Clinical training exceeding three years under the supervision by certified instructors; 2) a logbook with a list of required case experiences signed by supervisors; 3 ) passage of a written examination; 4) passage of an oral examination; and 5) submission of case reports. For JSPN certification as a psychiatry specialist, trainees are required to experience at least two CAP cases (versus minimum 10 cases of schizophrenia, 5 of mood disorders, 4 of organic psychosis, 5 of neurosis and stress related disorders) and to submit one CAP case report.

Fifty-eight percent of the instructors for the JSPN psychiatry specialist system are based in private psychiatric hospitals, $14 \%$ in clinics, $15 \%$ in university hospitals, and $12 \%$ in public hospitals. ${ }^{62}$ In terms of age, $10 \%$ of the instructors are in their 30 's, $33 \%$ in their 40 's, $33 \%$ in their 50 's, $18 \%$ in their 60 's, $5 \%$ in their 70's, and 1\% in their 80's.

In a survey on child and adolescent psychiatry practice, early-career psychiatrists in Japan indicated that, notwithstanding moderate interest in CAP (5.34 on a scale of 1-9), they had insufficient experience with (3.05) and knowledge of (3.43) CAP. ${ }^{1}$ Surveys of CAP interest indicated a decline in interest before (33.3\% interest) and after (20.5\%) one-year clinical training in general psychiatry and low ratings of CAP experience (3.46 on a 6-point scale). ${ }^{2,64}$ Surveys of clinical experience rated child and adolescent psychiatry lower than the other specialties (2.8 on a 5-point scale versus 3.2 to 4.0 for other areas, including schizophrenia, mood disorders, substance and alcohol, organic psychosis, neurosis and stress related disorders, and personality disorders). ${ }^{61}$ Overall, surveys found that during ordinary psychiatry training, psychiatric trainees will experience insufficient CAP cases. Furthermore, senior/supervising psychiatrists might need to teach in all psychiatric fields including CAP, even if they did not experience enough CAP cases in their own training or practice. It appears that psychiatry training with adequate CAP cases would be necessary to attract more early-career psychiatrists to CAP.

From the point of view of clinical education, it is important to think not only about training for young staff, but also about lifelong education (also known as CPE or continuous professional education) for practicing physicians. Currently, one can update one's certification only through attending congress meetings, which occur only five times in five years. The current Japanese training system has challenges and inade- 
quacies both in the training of new specialists and in lifelong education.

\section{COMPARISON WITH OTHER MODELS FOR CHILD AND ADOLESCENT PSYCHIATRIC TRAINING}

Child and adolescent psychiatry has been established as a clinical subspecialty in the US, where it constitutes a major part of psychiatric practice. Systematic education and training and research opportunities are also widely available in this specialty. In the US, pathways towards child and adolescent psychiatric certification include traditional training programs ( 3 years of general psychiatry plus 2 years of CAP), integrated training programs ( 5 years of training in general psychiatry and CAP at the same time), triple-board programs (2 years of pediatrics, 1.5 years of general psychiatry, and 1.5 years of CAP), and post-pediatric portal programs (3 years of training in general psychiatry and CAP following completion of pediatrics residency). Required experiences for traditional CAP training include: didactic sessions, faculty preceptorship (2 hours, including 1 individual), formulation, pediatric neurology, developmental disorders, psychiatric emergencies, acutely/severely disturbed children and adolescents (4-10 months), consultation (medical, school, and legal), community systems, normal development, and outpatients from each age group (>1 year).

As reviewed by Mian et al., ${ }^{65}$ child and adolescent psychiatry training and teaching in Europe ${ }^{66}$ and the United States has increasingly become more standardized. In the US, as specified by the Accreditation Council for Graduate Medical Education (ACGME) ${ }^{67}$ all CAP training programs must incorporate training and assessment in six major competency areas: patient care, medical knowledge, practice-based learning and improvement, interpersonal and communication skills, professionalism, and systems-based practice. ${ }^{68}$ In Europe, notwithstanding variability in specific training pathways across countries, ${ }^{69}$ the European Union of Medical Specialists (UEMS) has outlined a standard CAP curriculum that includes a minimum length of three years, certain basic competency goals, and an approval and auditing process by relevant government authorities responsible for education and training. ${ }^{70}$

In response to the many burdens that still need to be overcome in providing adequate child mental health care, important initiatives in US are: 1) improving the assessment of and recognition of mental health needs in children, 2) promoting scientifically-proven and evidence based interventions, and 3) increasing access to and coordination of quality mental health care services. Furthermore, in response to the increased emphasis on quality and accessibility of care within graduate medical education (the Clinical Learning Environment Review, or CLER initiative), US child and adolescent psychiatric training programs have adopted: quality improvement programs with ongoing feedback about clinical outcomes, standardized curricula that teach and evaluate specific competencies using progressive methods such as problem-based learning or $\mathrm{PBL},{ }^{71-74}$ and training experiences in telepsychiatry and primary care integration models. ${ }^{75}$

\section{UPCOMING NEW MOVEMENT OF CAP IN JAPAN AND OTHER RECOMMENDED SOLUTIONS}

To solve current problems in child and adolescent psychiatry in Japan, we advocate for the development and establishment of a more standardized child and adolescent psychiatry training system that is akin to what exists in the US and that teaches and evaluates according to specific competencies.

Overall, the certification system will become more standardized, and a new semi-official license system will start in 2018. In 2011, the Investigative Commission for Medical Specialist was established in the Ministry of Health, Labor and Welfare. In 2014, the Japanese Medical Specialty Board was established, and now they are programming a new training system for every medical discipline, including child and adolescent mental health (CAMH). This programming will include not only initial certification but also lifelong education.

In collaboration with the Japanese Medical Specialty Board (JMSB), which includes psychiatry as one of its 19 approved specialties, the JSPN will initiate new post-graduate psychiatry training in 2018. To harmonize this significant movement in medicine, four CAP-associated academic societies, the JSCAP, JSAP, JSPPN, and JSPP, will unify their respective specialist certification systems to one official specialist certification: a

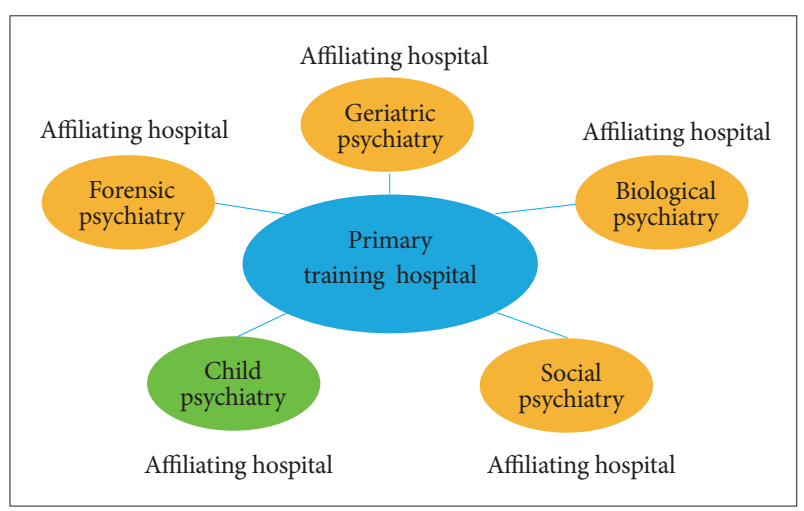

Figure 3. In the new specialty training system starting in 2017, training hospitals make a group to provide a better training program. The union of hospitals with various subspecialties will make the training program substantial and increase the exposure to CAP cases. CAP: child and adolescent psychiatry. 
specialist for child mental health. In the transitional period, applicants who have society-specific specialist certification and JSPN specialist accreditation or accreditation by the Japan Pediatric Society (JPS) can take lectures followed by written and oral examinations in order to be certified as a CAP specialist. This will be the great first step toward establishment of a uniform training program in CAP.

In the upcoming new training system, several teaching hospitals will create an alliance to educate psychiatry residents (Figure 3). Clinical training by rotating through various teaching hospitals and psychiatry clinics will increase the opportunities to be exposed to child cases with mental and/or developmental problems. We propose that the training consortium model will raise the standards of clinical practice and improve recruitment into the specialty. An increased workforce will, in turn, increase the availability of specialized clinical services that are currently in short supply (including inpatient child and adolescent psychiatric care) and will be available to teach and train subsequent generations of physicians.

Through standardizing care and education and ultimately improving workforce, we can raise the quality of mental health services. But is doing so enough? The problem of "lack of information" is still left. We propose to create a system to transparently share our treatment results to the public and government. The public are generally unaware of treatment efficacy and should be provided with the appropriate information. To document technical improvement, appropriate comparisons between therapists or clinics should be made. ${ }^{56}$

Mental illness is treatable, and the best outcomes occur with early identification and intervention. The tragic and costly consequences of unidentified and untreated mental illness in youth can be avoided by taking timely evidence based actions in partnership with others.

\section{CONCLUSION}

Despite increasing social demands, Japan has been facing a serious shortage of child and adolescent psychiatry (CAP) specialists. In this paper, we reviewed the current status of CAP training system in Japan. To avoid tragic consequences of unidentified and untreated mental illness in youth, we need to provide enough CAP workforce and appropriate accessibility to mental health services in partnership with the educational and child welfare systems. We advocate for the establishment and development of a standardized CAP training system that is similar to what exists in the US. In 2018, the Japanese Medical Specialty Board will initiate new specialty training system including psychiatry. The new movement in general psychiatry will accelerate the development of CAP training system.

\section{REFERENCES}

1. Tateno M, Uchida N, Kikuchi S, Kawada R, Kobayashi S, Nakano W, et al. The practice of child and adolescent psychiatry: a survey of earlycareer psychiatrists in Japan. Child Adolesc Psychiatry Ment Health 2009;3:30.

2. Tateno M, Kato T, Nakano W, Teo AR, Nakagawa A, Miyajima K, et al. Attitudes of early-career psychiatrists in Japan toward child and adolescent psychiatry and their career decision. Psychiatry Clin Neurosci 2010;64:199-201.

3. Agnew-Blais JC, Polanczyk GV, Danese A, Wertz J, Moffitt TE, Arseneault L. Evaluation of the persistence, remission, and emergence of attention-deficit/hyperactivity disorder in young adulthood. JAMA Psychiatry 2016;73:713-720.

4. Kim YS, Leventhal BL, Koh YJ, Fombonne E, Laska E, Lim EC, et al. Prevalence of autism spectrum disorders in a total population sample. Am J Psychiatry 2011;168:904-912.

5. Network AaDDMA. Community Report On Autism. The Centers for Disease Control and Prevention. Washington, D.C.: United States Department of Health and Human Services; 2016.

6. Autism and Developmental Disabilities Monitoring (ADDM) Network. Community Report On Autism. The Centers for Disease Control and Prevention. Washington, D.C.: United States Department of Health and Human Services; 2016.

7. Kurita H. Disorders of the autism spectrum. Lancet 2006;368:179-181.

8. Kawamura Y, Takahashi O, Ishii T. Reevaluating the incidence of pervasive developmental disorders: impact of elevated rates of detection through implementation of an integrated system of screening in Toyota, Japan. Psychiatry Clin Neurosci 2008;62:152-159.

9. Tateno M, Kikuchi S, Uehara K, Fukita K, Uchida N, Sasaki R, et al. Pervasive developmental disorders and autism spectrum disorders: are these disorders one and the same? Psychiatry Investig 2011;8:67-70.

10. WHO. The World Health Report 2001. Geneva: World Health Organization; 2001.

11. WHO. 10 Facts on Mental Health. Geneva: World Health Organization; 2011.

12. Rush AJ, Bernstein IH, Trivedi MH, Carmody TJ, Wisniewski S, Mundt JC, et al. An evaluation of the quick inventory of depressive symptomatology and the hamilton rating scale for depression: a sequenced treatment alternatives to relieve depression trial report. Biol Psychiatry 2006; 59:493-501.

13. Husain MM, Rush AJ, Sackeim HA, Wisniewski SR, McClintock SM, Craven N, et al. Age-related characteristics of depression: a preliminary STAR*D report. Am J Geriatr Psychiatry 2005;13:852-860.

14. Zisook S, Lesser I, Stewart JW, Wisniewski SR, Balasubramani GK, Fava M, et al. Effect of age at onset on the course of major depressive disorder. Am J Psychiatry 2007;164:1539-1546.

15. Copeland WE, Angold A, Shanahan L, Costello EJ. Longitudinal patterns of anxiety from childhood to adulthood: the Great Smoky Mountains Study. J Am Acad Child Adolesc Psychiatry 2014;53:21-33.

16. Schneider M, Debbane M, Bassett AS, Chow EW, Fung WL, van den Bree M, et al. Psychiatric disorders from childhood to adulthood in 22q11.2 deletion syndrome: results from the International Consortium on Brain and Behavior in 22q11.2 Deletion Syndrome. Am J Psychiatry 2014;171:627-639.

17. Kasparek T, Theiner P, Filova A. Neurobiology of ADHD from childhood to adulthood: findings of imaging methods. J Atten Disord 2015;19:931943.

18. Lewinsohn PM, Seeley JR, Buckley ME, Klein DN. Bipolar disorder in adolescence and young adulthood. Child Adolesc Psychiatr Clin N Am 2002;11:461-475, vii.

19. Rutter M. Relationships between mental disorders in childhood and adulthood. Acta Psychiatr Scand 1995;91:73-85.

20. McEwan K, Waddell C, Barker J. Bringing children's mental health "out of the shadows". CMAJ 2007;176:471-472. 
21. Kessler RC, Berglund P, Demler O, Jin R, Merikangas KR, Walters EE. Lifetime prevalence and age-of-onset distributions of DSM-IV disorders in the National Comorbidity Survey Replication. Arch Gen Psychiatry 2005;62:593-602.

22. Min JA, Lee CU, Lee C. Mental health promotion and illness prevention: a challenge for psychiatrists. Psychiatry Investig 2013;10:307-316.

23. Bertolote J, McGorry P. Early intervention and recovery for young people with early psychosis: consensus statement. Br J Psychiatry Suppl 2005;48:s116-s119.

24. McGorry PD, Killackey E, Yung A. Early intervention in psychosis: concepts, evidence and future directions. World Psychiatry 2008;7:148156.

25. Aboujaoude E. Problematic internet use: an overview. World Psychiatry 2010;9:85-90.

26. Yen CF, Yen JY, Ko CH. Internet addiction: ongoing research in Asia. World Psychiatry 2010;9:97.

27. Kim NR, Hwang SS, Choi JS, Kim DJ, Demetrovics Z, Kiraly O, et al. Characteristics and psychiatric symptoms of internet gaming disorder among adults using self-reported DSM-5 criteria. Psychiatry Investig 2016;13:58-66.

28. Lee CS, McKenzie K. Socioeconomic and geographic inequalities of internet addiction in Korean adolescents. Psychiatry Investig 2015;12:559562.

29. Yang SJ, Stewart R, Lee JY, Kim JM, Kim SW, Shin IS, et al. Prevalence and correlates of problematic internet experiences and computer-using time: a two-year longitudinal study in Korean school children. Psychiatry Investig 2014;11:24-31.

30. Kawanishi C, Aruga T, Ishizuka N, Yonemoto N, Otsuka K, Kamijo Y, et al. Assertive case management versus enhanced usual care for people with mental health problems who had attempted suicide and were admitted to hospital emergency departments in Japan (ACTION-J): a multicentre, randomised controlled trial. Lancet Psychiatry 2014;1: 193-201.

31. Cabinet Office Government of Japan. Statistics of suicide by National Police Agency, Japan. Tokyo: Cabinet Office, Government of Japan; 2016.

32. Kawabe K, Horiuchi F, Ochi M, Oka Y, Ueno S. Suicidal ideation in adolescents and their caregivers: a cross sectional survey in Japan. BMC Psychiatry 2016;16:231.

33. Matsubayashi T, Ueda M, Yoshikawa K. School and seasonality in youth suicide: evidence from Japan. J Epidemiol Community Health 2016;70:1122-1127.

34. Naito A. Internet suicide in Japan: implications for child and adolescent mental health. Clin Child Psychol Psychiatry 2007;12:583-597.

35. Wright MF, Yanagida T, Aoyama I, Dedkova L, Li Z, Kamble SV, et al. Differences in attributions for public and private face-to-face and cyber victimization among adolescents in China, Cyprus, the Czech Republic, India, Japan, and the United States. J Genet Psychol 2017;178:1-14.

36. Honjo S, Nishide T, Niwa S, Sasaki Y, Kaneko H, Inoko K, et al. School refusal and depression with school inattendance in children and adolescents: comparative assessment between the Children's Depression Inventory and somatic complaints. Psychiatry Clin Neurosci 2001;55: 629-634.

37. Inoue K, Tanii H, Nishimura Y, Masaki M, Nishida A, Kajiki N, et al. Current state of refusal to attend school in Japan. Psychiatry Clin Neurosci 2008;62:622.

38. Honjo S, Sasaki Y, Kaneko H, Tachibana K, Murase S, Ishii T, et al. Study on feelings of school avoidance, depression, and character tendencies among general junior high and high school students. Psychiatry Clin Neurosci 2003;57:464-471.

39. Borovoy A. Japan's hidden youths: mainstreaming the emotionally distressed in Japan. Cult Med Psychiatry 2008;32:552-576.

40. Nishida A, Sugiyama S, Aoki S, Kuroda S. Characteristics and outcomes of school refusal in Hiroshima, Japan: proposals for network therapy. Acta Med Okayama 2004;58:241-249.

41. MECSST. Report on School Withdrawals. Tokyo: Ministry of Educa- tion, Culture, Sports, Science and Technology; 2014.

42. Teo AR, Fetters MD, Stufflebam K, Tateno M, Balhara Y, Choi TY, et al. Identification of the hikikomori syndrome of social withdrawal: psychosocial features and treatment preferences in four countries. Int J Soc Psychiatry 2015;61:64-72.

43. Tateno M, Park TW, Kato TA, Umene-Nakano W, Saito T. Hikikomori as a possible clinical term in psychiatry: a questionnaire survey. BMC Psychiatry 2012;12:169.

44. Kato TA, Tateno M, Shinfuku N, Fujisawa D, Teo AR, Sartorius N, et al. Does the 'hikikomori' syndrome of social withdrawal exist outside Japan? A preliminary international investigation. Soc Psychiatry Psychiatr Epidemiol 2012;47:1061-1075.

45. Koyama A, Miyake Y, Kawakami N, Tsuchiya M, Tachimori H, Takeshima T, et al. Lifetime prevalence, psychiatric comorbidity and demographic correlates of "hikikomori" in a community population in Japan. Psychiatry Res 2010;176:69-74.

46. Kato TA, Shinfuku N, Sartorius N, Kanba S. Are Japan's hikikomori and depression in young people spreading abroad? Lancet 2011;378:1070.

47. Teo AR, Gaw AC. Hikikomori, a Japanese culture-bound syndrome of social withdrawal?: a proposal for DSM-5. J Nerv Ment Dis 2010;198:444449.

48. Teo AR. A new form of social withdrawal in Japan: a review of hikikomori. Int J Soc Psychiatry 2010;56:178-185.

49. Krieg A, Dickie JR. Attachment and hikikomori: a psychosocial developmental model. Int J Soc Psychiatry 2013;59:61-72.

50. Hirota T, Guerrero A, Sartorius N, Fung D, Leventhal B, Ong SH, et al. Child and adolescent psychiatry in the Far East. Psychiatry Clin Neurosci 2015;69:171-177.

51. MECSST. White Paper on Education, Culture, Sports, Science and Technology. Tokyo: Ministry of Education, Culture, Sports, Science and Technology; 2010.

52. McClure M, Shirataki S. Child psychiatry in Japan. J Am Acad Child Adolesc Psychiatry 1989;28:488-492.

53. Kitagawa Y, Shimodera S, Togo F, Okazaki Y, Nishida A, Sasaki T. Suicidal feelings interfere with help-seeking in bullied adolescents [corrected]. PLoS One 2014;9:e106031.

54. Ogura M, Okada K, Hamada S, Asaga R, Honjo S. Ijime in Japan. Int J Adolesc Med Health 2012;24:69-76.

55. MECSST. Report on Problems of Students. Tokyo: Ministry of Education, Culture, Sports, Science and Technology; 2016.

56. Inagaki T. Current trial of child and adolescent mental health services in Shiga prefecture, Japan. Psychiatry (in Japanese) 2016;28:332-337.

57. Heflinger CA, Hinshaw SP. Stigma in child and adolescent mental health services research: understanding professional and institutional stigmatization of youth with mental health problems and their families. Adm Policy Ment Health 2010;37:61-70.

58. Pescosolido BA, Perry BL, Martin JK, McLeod JD, Jensen PS. Stigmatizing attitudes and beliefs about treatment and psychiatric medications for children with mental illness. Psychiatr Serv 2007;58:613-618.

59. Tsuchiya KJ, Takei N. Focus on psychiatry in Japan. Br J Psychiatry 2004; 184:88-92.

60. Tan S, Fung D, Hung SF, Rey J. Growing wealth and growing pains: child and adolescent psychiatry in Hong Kong, Malaysia and Singapore. Australas Psychiatry 2008;16:204-209.

61. Umene-Nakano W, Uchida N, Kato T, Tateno M, Matsumoto R, Nakamura J. [Views on the new psychiatric specialist certification system from the perspective of those experiencing the postgraduate psychiatric training system in Japan]. Seishin Shinkeigaku Zasshi 2011;113:271276.

62. Yamauchi T. Views on the new psychiatric specialist certification system from the perspective of those experiencing the postgraduate. Seishin Shinkeigaku Zasshi 2016;118:287-303.

63. Kojima T. Training system and the current status of psychiatry specialist certification system. Seishin Shinkeigaku Zasshi 2011;113:250-257.

64. Miyajima K, Fujisawa D, Nakagawa A, Kato T. [Psychiatric training at 
university hospitals--a viewpoint of the first psychiatrists who were trained at the latter part of the new system]. Seishin Shinkeigaku Zasshi 2007;109:1039-1044.

65. Mian AI, Milavic G, Skokauskas N. Child and adolescent psychiatry training: a global perspective. Child Adolesc Psychiatr Clin N Am 2015;24:699-714.

66. Simmons M, Barrett E, Wilkinson P, Pacherova L. Trainee experiences of Child and Adolescent Psychiatry (CAP) training in Europe: 20102011 survey of the European Federation of Psychiatric Trainees (EFPT) CAP working group. Eur Child Adolesc Psychiatry 2012;21:433-442.

67. Swick S, Hall S, Beresin E. Assessing the ACGME competencies in psychiatry training programs. Acad Psychiatry 2006;30:330-351.

68. Ascherman LI, Lamps C. The child and adolescent psychiatry match: problems and potential solutions. Acad Psychiatry 2005;29:8-13.

69. Karabekiroglu K, Dogangun B, Herguner S, von Salis T, Rothenberger A. Child and adolescent psychiatry training in Europe: differences and challenges in harmonization. Eur Child Adolesc Psychiatry 2006;15: 467-475.
70. Rothenberger A. The training logbook of UEMS section/board on child and adolescent psychiatry (CAPP) progress concerning European harmonization. Eur Child Adolesc Psychiatry 2001;10:211-213.

71. Skokauskas N, Guerrero AP, Hanson MD, Coll X, Paul M, Szatmari P, et al. Implementation of problem-based learning in child and adolescent psychiatry: shared experiences of a special-interest study group. Acad Psychiatry 2011;35:249-251.

72. Skokauskas N, Doody B, Gallagher L, Lawlor M, Moran T, Fitzgerald $\mathrm{M}$, et al. Problem-based learning in child and adolescent psychiatry at Trinity College, Dublin, Ireland. Acad Psychiatry 2012;36:335-339.

73. Azer SA, Peterson R, Guerrero AP, Edgren G. Twelve tips for constructing problem-based learning cases. Med Teach 2012;34:361-367.

74. Guerrero AP. Mechanistic case diagramming: a tool for problem-based learning. Acad Med 2001;76:385-389.

75. Alicata D, Schroepfer A, Unten T, Agoha R, Helm S, Fukuda M, et al. Telemental health training, team building, and workforce development in cultural context: the Hawaii experience. J Child Adolesc Psychopharmacol 2016;26:260-265. 